\title{
S3-Leitlinie Sedierung in der gastrointestinalen Endoskopie: Kurzfassung apparativer, personeller und struktureller Voraussetzungen und juristische Implikationen bei der Umsetzung
}

\author{
Short Version S3 Guideline Sedation for Gastrointestinal Endoscopy und medicolegal implications
}

Autoren

Institute
A. Riphaus ${ }^{1}$, H. Bitter ${ }^{2}$

Medizinische Universitätsklinik, Knappschaftskrankenhaus, Ruhr-Universität Bochum

2 Kanzlei Ehlers, Ehlers \& Partner
Schlüsselwörter

- Sedierung

- Leitlinie

- juristische Aspekte

Key words

- sedation

- guideline

- medicolegal aspects

eingereicht 26.10 .2011

akzeptiert 21.1.2012

Bibliografie

Dol http://dx.doi.org/

10.1055/s-0031-1299290

Z Gastroenterol 2012; 50: $407-$

410 ๑ Georg Thieme Verlag KG Stuttgart · New York .

ISSN 0044-2771

\section{Korrespondenzadresse}

PD Dr. Andrea Riphaus

Medizinische Universitätsklinik, Knappschaftskrankenhaus

Bochum-Langendreer

In der Schornau 23-25

44892 Bochum

Tel.: ++ 49/2 34/2990

Fax: ++ 49/2 34/2993409

ariphaus@web.de

\section{Zusammenfassung}

\section{$\nabla$}

Durch die Veröffentlichung der ersten bundesdeutschen S3-Leitlinie zur Sedierung in der gastrointestinalen Endoskopie hat das Thema Sedierung neuerlich sowohl bei Ärzten und Assistenzpersonal als auch in der Laienpresse an Interesse gewonnen. Es bleibt weiterhin Gegenstand vieler, oft auch kontroverser Diskussionen. Diese Diskussion rührt vor allem aus der Tatsache, dass die Leitlinie zwar einen strukturierten Überblick über die bereits existierenden Anforderungen an strukturelle wie persönliche Qualifikation, notwendige Personalressourcen, Standards zur adäquaten Aufklärung über die Sedierung sowie die Risikostratifizierung der Patienten, die mit Veröffentlichung der Leitlinie eine vermehrte Aufmerksamkeit erlangte.

\section{Einführung}

$\nabla$

Durch die Veröffentlichung der ersten bundesdeutschen S3-Leitlinie zur Sedierung in der gastrointestinalen Endoskopie [1] hat das Thema Sedierung neuerlich sowohl bei Ärzten, Assistenzpersonal als auch in der Laienpresse an Interesse gewonnen. Es bleibt weiterhin Gegenstand vieler, oft auch kontroverser Diskussionen. Diese Diskussion rührt vor allem aus der Tatsache, dass die Leitlinie zwar einen strukturierten Überblick über die bereits existierenden Anforderungen an strukturelle wie persönliche Qualifikation, notwendige Personalressourcen, Standards zur adäquaten Aufklärung über die Sedierung sowie die Risikostratifizierung der Patienten, die mit Veröffentlichung der Leitlinie eine vermehrte Aufmerksamkeit erlangte, gibt, sie zeigt jedoch auch Defizite in deren Umsetzung, die hauptsächlich durch eine fehlende Bereitschaft vieler Krankenhausleitungen verursacht sind, die hierfür notwendigen Umstrukturierungen und ggf. finanziellen Ressourcen bereitzustellen, zumal eine Erstattung

\section{Abstract \\ $\nabla$}

With the publication of the first German S3 guideline on sedation for gastrointestinal endoscopy, the topic sedation has recently attracted the interest not only of physicians and medical assistants but also of the general public. It remains the topic of many, often controversial, discussions. This discussion arises mainly because of the fact that with the guideline - although giving a structured survey of the already existing requirements on structural as well as personnel qualifications - more emphasis is placed on the necessary personnel resources, standards for adequate patient information on the sedation, and risk stratification for the patient with the publication of the guideline.

der im Rahmen der Sedierung erbrachten und durch die Leitlinie erforderlichen Mehrkosten bis heute nicht umgesetzt ist.

Einen neuen Aspekt stellt hierbei auch die durch ein juristisches Fachgutachten von Prof. Dr. Dr. Alexander Ehlers und Dr. Horst Bitter (s. Homepage der DGVS) und verschiedene ärztliche Fachgruppen abgesicherte und befürwortete Delegation der Sedierung mit Propofol an nicht ärztliches Assistenzpersonal (sog. „nurse administered propofol sedation“, kurz NAPS), innerhalb klar definierter Grenzen, dar. Besonders hervorzuheben ist hierbei, dass diese Empfehlungen unter Einbindung sämtlicher relevanter Berufsgruppen (Gastroenterologen, Anästhesisten, Chirurgen, endoskopisches Assistenzpersonal, Juristen, Patientenselbsthilfegruppen) erstellt und verabschiedet wurde, was in dieser Form weltweit einmalig ist.

In dem folgenden Beitrag werden zunächst die wesentlichen aktuellen Empfehlungen der Leitlinie (kursivgedruckt) mit den entsprechenden Leitlinien-Kommentaren zusammengefasst. Im Anschluss folgt eine juristische Stellungnahme zu 
möglichen Konsequenzen für behandelnde Ärzte sowie Entscheidungsträger von Krankenhäusern, Medizinischen Versorgungszentren und Praxen bei fehlender Umsetzung der geforderten Mindeststandards.

\section{Patientenvorbereitung und Monitoring}

$\nabla$

Nach entsprechender Aufklärung des Patienten und erfolgter Risikoevaluation für mögliche Komplikationen, die während der Sedierung auftreten, bedarf es der Vorbereitung des Patienten für die Sedierung und nachfolgende Endoskopie. „Bei Patienten mit höherem Risikoprofil soll die Hinzuziehung eines Anästhesisten erwogen werden, dazu gehören: hohe ASA-Klassifikation (III-IV) und schwierige endoskopische Eingriffe oder wenn durch pathologischanatomische Besonderheiten des Patienten ein erhöhtes Risiko für eine Atemwegsbehinderung während des Eingriffs gegeben ist ( $z$. B. bei kraniofazialen Missbildungen, Tumoren des Zungengrunds, Larynx oder Hypopharynx, massiv eingeschränkter Beweglichkeit der HWS, massiv eingeschränkter Mundöffnung $<3 \mathrm{~cm}$, MallampattiStadien 3 oder 4 bzw. eingeschränkter Kehlkopf-Kinnspitzen Abstand von weniger als $6 \mathrm{~cm}$ ).“

Die „Voraussetzung für eine Sedierung ist ein permanenter intravenöser Zugang“. Zur Reduktion von sedierungsassoziierten Hypoxämien, die während der Untersuchung auftreten können, „sollen sedierte Patienten prophylaktisch Sauerstoff über eine Nasensonde erhalten“. „Zu den notwendigen Maßnahmen des Monitoring gehören die Pulsoxymetrie und die Blutdruckmessung. Bei Patienten mit schwerer Herzerkrankung sollte darüber hinaus eine EKG-Registrierung erfolgen.“

\section{Strukturqualität - persönliche - personelle - apparati- ve Voraussetzungen}

Sowohl der diagnostische oder therapeutische Eingriff als auch die Sedierung sind eigenständige medizinische Verfahren. Führt ein Arzt den diagnostischen oder therapeutischen Eingriff und gleichzeitig das Sedierungsverfahren durch, übernimmt er nicht nur für den Eingriff, sondern auch für die Sedierung und/oder die Analgesie einschließlich der Überwachung und gegebenenfalls Wiederherstellung vitaler Funktionen die volle Verantwortung. „Er soll in der Anwendung von Sedativa und damit in Kenntnis, Erkennung und Behandlung der zu erwartenden Nebenwirkungen, einschließlich der kardiopulmonalen Reanimation, dem Freimachen/Freihalten eines durchgängigen Luftweges, der Intubation und manuelle Beatmung speziell geschult sein und sie beherrschen.“

Hierbei sind spezielle Kenntnisse in Theorie und Praxis der Sedierung und/oder Analgesie nicht nur für den Arzt, sondern auch für das ihn unterstützende nicht ärztliche Personal notwendig. „Im Rahmen der Qualitätssicherung soll ein speziell auf die Sedierung ausgerichtetes Training für Ärzte und nicht-ärztliches Assistenzpersonal durchgeführt werden.“

„Die Qualifikation des ärztlichen sowie des nicht ärztlichen Personals, welches an der Durchführung der Sedierung, Überwachung und Nachsorge beteiligt ist, soll durch periodische Teilnahme an strukturierten Fortbildungscurricula sichergestellt werden. Neben theoretischen Kenntnissen werden auch praktische Fähigkeiten inkl. Komplikationsmanagement (z. B. im Simulatormodell) in diesen Curricula vermittelt. Hierbei sollte insgesamt ein gemeinsames
Training des gesamten Untersucherteams (ärztliches und nichtärztliches Personal) bevorzugt werden. “

Um diesen Anforderungen Rechnung zu tragen, sind von der DGVS neben einem strukturierten 1-Tages-Teamtraining-Refresherkurs als auch in Zusammenarbeit mit der DEGEA ein 3-Tages-Basiskurs für Assistenzpersonal etabliert worden, damit die Ausbildung zur Qualitätssicherung bundesweit vereinheitlicht werden kann.

Weiterhin kann ein Arzt nicht in Personalunion zur gleichen Zeit den invasiven Eingriff durchführen und die Sedierung und/oder das Analgesieverfahren überwachen.

Folgerichtig soll daher nicht der den Eingriff durchführende Arzt, sondern eine speziell geschulte Person für das Sedierungsverfahren und die Überwachung der Vitalfunktionen verantwortlich sein. „Der endoskopierende Arzt ist während der Durchführung der Endoskopie in aller Regel nicht in der Lage, den Vitalfunktionen des Patienten die notwendige Aufmerksamkeit zu schenken. Es ist daher für jede Endoskopie unter Sedierung erforderlich, dass neben dem endoskopierenden Arzt und seiner Endoskopieassistenz eine weitere Person, die nicht in die Endoskopie involviert ist, diese Aufgabe zuverlässig wahrnimmt. Diese qualifizierte Person soll in der Überwachung von Patienten, die Sedativa, Hypnotika und/oder Analgetika erhalten, speziell und nachweislich geschult und erfahren sein. Wann immer der Patient ein erhöhtes Risiko aufweist oder ein langwieriger und aufwendiger Eingriff zu erwarten ist, soll ein zweiter, entsprechend qualifizierter Arzt zugegen sein, der ausschließlich die Durchführung und Überwachung der Sedierung sicherstellt."

Die die Sedierung überwachende Person darf in dieser Zeit keine anderen Aufgaben wahrnehmen. Wann diese ärztliche Person durch qualifiziertes, speziell geschultes nicht ärztliches Personal ersetzt werden kann, ist im jeweiligen Einzelfall unter Berücksichtigung der Struktur der Arbeitsstätte, des Zustands des Patienten und der Komplexität der Intervention vor Ort zu beurteilen und zu verantworten. Es obliegt der Sorgfaltspflicht des den Eingriff durchführenden Arztes, sich zu vergewissern, dass diese Person ausreichend qualifiziert und in der Lage ist, ihre Aufgaben auch adäquat zu erfüllen.

„Bei einfachen endoskopischen Untersuchungen und risikoarmen Patienten soll die Sedierung durch den entsprechend qualifizierten Arzt eingeleitet werden und kann anschließend von einer entsprechend ausgebildeten und erfahrenen Person überwacht werden. Die die Sedierung überwachende Person darf in dieser Zeit keine anderen Aufgaben wahrnehmen. Propofol kann von einer entsprechend ausgebildeten und erfahrenen Person, die ausschließlich mit dieser Aufgabe betraut ist, auf ärztliche Anordnung während der Untersuchung verabreicht werden.

Unter entsprechenden Voraussetzungen ist NAPS sicher und effizient bei Patienten mit ASA- Klassifikation I-III. „Bei Patienten mit erhöhtem Risikoprofil oder komplexen, lang dauernden therapeutischen Eingriffen, die einer tieferen Sedierung bedürfen und dadurch mit einem erhöhten Sedierungsrisiko assoziiert sein können, soll ein zweiter, intensivmedizinisch erfahrener, Arzt die Sedierung durchführen.“

Auch die Dokumentation ist ein essentieller Bestandteil der Patientenfürsorge und soll während aller Phasen der Untersuchung durchgeführt werden.

Hierzu zählen

- die prä-interventionelle Einschätzung des Patienten

- die Aufklärung des Patienten

- das Monitoring während der Untersuchung

$\checkmark$ die Patientenerholung

- die Patientenentlassung 
Idealerweise sollte hier ein standardisierter Dokumentationsbogen verwandt werden, da hierdurch die Einhaltung der Dokumentation verbessert werden kann. Ensprechende individuell modifizierbare Formulare wurden als Worddatei auf der Homepage der DGVS und der DEGEA zum Abrufen hinterlegt.

Speziell für das Monitoring während der Untersuchung gilt: Die Akte oder das Dokumentationsblatt soll eine zeitabhängige Dokumentation der Vitalparameter (Herzfrequenz und Blutdruck), der verwendeten Medikamente mit Namen und Dosierung, sowie der Gabe intravenöser Flüssigkeit enthalten und Angaben darüber machen, ob und in welcher Flussrate der Patient Sauerstoff erhalten hat. Idealerweise sollen periodisch auch der Sedierungsgrad und Schmerzangaben des Patienten dokumentiert werden.

\section{Postinterventionelle Überwachung \\ $\nabla$}

„Die Überwachung des Patienten in der Aufwachphase soll durch entsprechend geschultes und qualifiziertes Personal vorgenommen werden."

Die entsprechende Person muss ständig im Aufwachbereich sein bzw. die Überwachungseinheit im Blickkontakt haben. Sie darf jedoch z.B. telefonieren oder Befunde abheften. Aus Gründen der Patientensicherheit sollen auch bereits wache Patienten bis zur Entlassung im Überwachungsbereich verweilen. Somit kann verhindert werden, dass der evtl. noch beeinträchtigte Patient eigenständig den Untersuchungsbereich verlässt. Der BGH hat laut Urteil vom 8.4.2003, Aktenzeichen: VI ZR 265/02, die Verbringung eines Patienten in einen Flurbereich, unmittelbar nach erfolgter Endoskopie unter Sedierung (Midazolam), beanstandet. Der Aufwachbereich muss als solcher definiert (und damit abgegrenzt) sein und der Patient unter ständiger Aufsicht stehen.

Die Problematik des Organisations-/Übernahmeverschuldens ergibt sich aus den allgemeinen Rechtsgrundsätzen, welche sich aus dem Zivil-, Straf- und Berufsrecht herleiten und wird im Nachfolgenden weiter erörtert.

\section{Juristische Implikationen und Konsequenzen \\ $\nabla$}

Juristen treten regelmäßig „auf den Plan“, wenn ein Patient zu Schaden gekommen ist. Dann stellt sich die Frage der Haftung. Im vorliegenden Zusammenhang ist damit zu erörtern, welche rechtliche Bedeutung der Rolle der S3-Leitlinie und den in ihr normierten Voraussetzungen in einem etwaigen - nachfolgend unterstelltem - Schadensfall zukommt.

Zivil- und strafgerichtliche Verfahren, die sich mit einer etwaigen Haftung von Ärzten, Pflegepersonal, Verwaltungsangestellten und Krankenhausträgern etc. auseinandersetzen, haben als zentralen Punkt - vereinfacht ausgedrückt - regelmäßig die Erörterung zum Gegenstand, ob ein Behandlungsfehler vorliegt oder nicht. Ob dies der Fall ist, bestimmt sich letztlich danach, ob im konkreten Einzelfall der medizinische Standard gewahrt wurde. Leitlinien kommt sicherlich eine Indizfunktion für den jeweiligen Stand der wissenschaftlichen Erkenntnis zu. Leitlinien können aber nicht mit einem medizinischen Standard gleichgesetzt werden. Letztlich sind Leitlinien Orientierungshilfen im Sinne von Handlungs- und Entscheidungskorridoren, von denen in begründeten Fällen abgewichen werden kann oder sogar muss. In einem etwaigen Prozess geben Leitlinien also dem regelmäßig vom Gericht beauftragten Sachverständigen kein Ergebnis vor. Aufgrund ihrer Indizwirkung muss der Sachverständige - wenn er im kon- kreten Einzelfall ein Abweichen von den Grundsätzen einer Leitlinie befürworten möchte - dies allerdings konkret begründen. Wenn man die vorstehend schon einmal kurz zusammengefassten Grundsätze nach der S3-Leitlinie noch einmal fokussiert, stellt man fest, dass

- die Einleitung stets durch einen Arzt mit entsprechender Qualifikation (zumindest intensiv-medizinische Erfahrung) zu erfolgen hat,

- nach Einleitung der Sedierung die Übergabe an nicht ärztliches, entsprechend qualifiziertes Personal möglich ist, sofern es sich um Nicht-Risikopatienten bzw. um einen einfachen Eingriff handelt und

- im Falle der Delegation der Überwachung an nicht ärztliches Personal erforderlichenfalls ein zweiter Arzt mit entsprechender Qualifikation unverzüglich hinzugezogen werden kann.

Nunmehr soll auf den eingangs erwähnten „Fall vor Gericht“ aus juristischer Sicht zurückgekommen werden. Welche Konsequenzen können sich für die beteiligten Personen ergeben, die an einer Behandlung mitgewirkt haben, bei der ein Patient zu Schaden gekommen ist. Allgemein ist festzustellen, dass zwischen einer zivil- und strafrechtlichen Verantwortlichkeit differenziert werden muss. Vereinfacht geht es im Zivilprozess um die Geltendmachung von finanziellen Schadensersatzforderungen aufgrund eines durch medizinische Behandlung eingetretenen gesundheitlichen Schadens. Ein etwaiger Behandlungsfehler birgt aber zudem das Risiko, dass eine oder mehrere Personen auch strafrechtlich zur Verantwortung gezogen werden. Hervorzuheben ist insoweit, dass die strafrechtliche Verantwortung stets von natürlichen Personen zu tragen ist, nicht aber von juristischen Personen (z. B. einer GmbH).

Bezogen auf die an dieser Stelle diskutierte S3-Leitlinie ist also in einem etwaigen „Fall vor Gericht“ zu prüfen, ob ein Patient aufgrund der Nichteinhaltung der in der S3-Leitlinie empfohlenen Standards zu Schaden gekommen ist oder nicht. Ein Behandlungsfehler wird dann anzunehmen sein, wenn ein Sachverständiger zu dem Ergebnis kommt, dass die Nichteinhaltung der in der S3-Leitlinie festgelegten Voraussetzungen im konkreten Fall die Annahme einer Verletzung des medizinischen Standards rechtfertigt. Folgt das Gericht dieser Einschätzung, würde dem Patienten in einem Zivilprozess Schadensersatz zugesprochen. Neben dem Zivilprozess kann es auch zu einem Strafverfahren kommen. Bspw. ist ein niedergelassener Gynäkologe wegen einer Sedierung mit Propofol, bei der es zu einer von ihm nicht beherrschten Reanimation kam, wegen fahrlässiger Tötung zu einer Freiheitsstrafe von 1 Jahr und 8 Monaten ohne Aussetzung der Freiheitsstrafe zu Bewährung verurteilt worden (Urteil des Amtsgerichts München vom 13.12.2005, Az. 824 Ds. 125, abgedruckt in ArztRecht 3/2007, S. 69 ff.). In dem vom Amtsgericht entschiedenen Fall stand dem Gynäkologen kein weiterer Arzt zur Überwachung der Atmung der Patientin zur Verfügung. Seine Praxis verfügte nicht über die für den Eingriff erforderliche apparative Ausstattung und er hatte keinen geeigneten Reanimationsmaßnahmen durchgeführt. Erst im Berufungsverfahren ist die Freiheitsstrafe zur Bewährung ausgesetzt worden (Urteil des Landgerichts München I vom 26.7.2006, Az. 16 Ns. 125, abgedruckt a.a. O.). In einer Einleitung zur Darstellung der beiden vorgenannten Urteile heißt es an der vorbenannten Fundstelle: „Die in Deutschland immer noch weitverbreitete Sorglosigkeit beim Einsatz zentral nervös wirkender Medikamente sollte spätestens mit diesem Urteil der Vergangenheit angehören. " Hervorzuheben ist, dass sich eine zivil- und strafrechtliche Verantwortlichkeit auch unter dem Gesichtspunkt des „Organisationsverschuldens“ ergeben kann. 
Denkbar wäre bspw., dass in der Leitlinie festgesetzte Standards in sachlicher und/oder personeller Hinsicht in einem konkreten Einzelfall nicht erfüllt sein könnten. Wenn ein vom Gericht bestellter Sachverständiger in einem etwaigen gerichtlichen Verfahren hieraus eine Verletzung des medizinischen Standards ableiten würde, könnte sich auch aus solchen organisatorischen Versäumnissen eine zivil- und/oder strafrechtliche Verantwortung ergeben. Anders als in einem Strafprozess kann sich in einem Zivilprozess eine unmittelbare Haftung des Krankenhausträgers ergeben. Ein Organisationsverschulden des Krankenhausträgers wurde beispielsweise vom OLG Stuttgart bejaht, als ein Anästhesist nach telefonischer Anforderung erst nach 20 - 25 min zur Verfügung stand (vgl. OLG Stuttgart, NJW 1993, Seite $2384 \mathrm{ff}$.). In strafrechtlicher Hinsicht kann sich in einer solchen Konstellation eine Verantwortung nicht nur der behandelnden Ärzte und des Pflegepersonals, sondern auch der für die Krankenhausorganisation verantwortlichen Personen - einschließlich der Entscheidungsträger - ergeben. Die Möglichkeit einer persönlichen strafrechtlichen Verantwortlichkeit wird oftmals dies zeigen Erfahrungen aus der Praxis - von Ärzten, Pflegern und den in der Verwaltung tätigen Personen bzw. Entscheidungsträgern nicht gesehen. In einer solchen Konstellation kann man sich regelmäßig nicht darauf berufen, aufgrund eines Arbeitsvertrags oder eines Beamtenverhältnisses im Auftrag des Arbeitgebers bzw. Dienstherrn tätig geworden zu sein. Anzumerken und hervorzuheben ist zudem, dass weder eine zivil- noch eine strafrechtliche Verantwortlichkeit im Haftungsfall mit der Begründung abgewendet werden kann, dass nicht ausreichend Gelder für die Wahrung des medizinischen Standards, der durch eine S3-Leitlinie indiziert sein kann, zur Verfügung stehen. Für die Bereitstellung ausreichender räumlicher Ressourcen (definierter Aufwach- und Überwachungsraum) sowie personeller Ressourcen (immer zur Sedierungsüberwachung qualifizierte dritte Person im Behandlungsraum, bei Hochrisikopatienten Anästhesist, schnelle Verfügbarkeit eines Anästhesisten) ist regelmäßig die jeweilige Krankenhausleitung zuständig. Im Falle der Nichteinhaltung von Standards können sich persönliche Verantwortlichkeiten für Ärzte, Pfleger und in der Verwaltung tätige Personen ergeben. Vorsorglich sollten Missstände bzw. Defizite dokumentiert werden, um nachhaltig für Beachtung Sorge zu tragen und - nicht zuletzt - um Verantwortlichkeiten zu klären. Die Darstellung juristischer Implikationen und Konsequenzen der Nichteinhaltung einer S3-Leitlinie ist komplex und umfassend. Sie können an dieser Stelle nur vereinfacht und verkürzt dargestellt werden. Abschließend sei empfohlen, dass die Einhaltung von S3-Leitlinien und der einschlägigen Fachinformationen vorsorglich gewahrt werden sollte, sofern nicht begründet im konkreten Einzelfall davon abgewichen werden kann.

\section{Propofol-Sedierung durch nicht ärztliches Assistenz- personal \\ $\nabla$}

Daten und aktuelle Rechtlage in Deutschland

Nach den ersten großen Studien zur Propofol-Bolus-Sedierung durch Assistenzpersonal im Rahmen der Koloskopie aus den
USA und der Schweiz an jeweils mehr als über 2000 Patienten $[2,3]$, bei denen neben einer kurzfristigen Maskenbeatmung in 0,2 \% der Fälle kein einziger Patient einer endotrachealen Intubation bedurfte, wurde dieses Verfahren auch bei uns in Deutschland, aufgrund abnehmender Personalressourcen im ärztlichen Bereich und notweniger Kostenreduktion für die einzelne Untersuchung bei steigendem Kostendruck, als alternatives Verfahren diskutiert. Um diese Sedierungsoption auch unter juristischen Aspekten für die Anwendung in Deutschland zu prüfen, wurde 2006 ein entsprechendes Rechtsgutachten durch die DGVS in Auftrag gegeben. Unter Berücksichtigung der zu diesem Zeitpunkt aktuellen Studiendaten und der geltenden Rechtsprechung in Deutschland kamen die Gutachter Prof. Dr. Dr. Alexander Ehlers und Dr. Horst Bitter zu dem Ergebnis, dass trotz der bestehenden Anwendungsbeschränkung des AMG zumindest „...die teilweise Übertragung der Applikation von Propofol an nicht-ärztliches Assistenzpersonal unter Gewährleistung einer sofortigen Präsenz eines zweiten in der Intensivmedizin erfahrenen Arztes oder eines Anästhesisten möglich.“ Erneut und weiterhin muss jedoch darauf hingewiesen werden, dass aufgrund der bestehenden deutschen Rechtslage (die Verabfolgung von Narkotika gilt als primäre ärztliche Aufgabe) eine „eigenständige“ bzw. „eigenverantwortliche“ Anwendung von Propofol durch Assistenzpersonal nicht möglich ist. Unabdingbar ist, dass der initial die Sedierung einleitende und dann an das Assistenzpersonal delegierende Arzt in jedem Einzelfall über den Patienten (z.B. Anamnese und Vor-Medikation) informiert sein muss, bevor er konkret die Delegation der Propofol-Gabe an das Assistenzpersonal vornimmt. Darüber hinaus trägt er für die Sedierung und die sich ergebenden Komplikationen die volle Verantwortung und muss sich im Einzelnen persönlich über die Qualifikation des Assistenzpersonals regelmäßig Gewissheit verschaffen. Neben bereits bestehenden Ausbildungscurricula für Assistenzpersonal, die in den USA bis zu 3 Wochen dauern und in der Schweiz aktuell von 6 Monaten auf noch immerhin 8 Wochen reduziert wurden, wurde nun auch erstmalig in Deutschland, mit dem 3-TagesCurriculum der DEGEA, ein strukturiertes Curriculum vorgestellt, um bundesweit eine standardisierte Ausbildung zu ermöglichen. Mittlerweile liegt eine gemeinsame Anerkennung dieser Kurse mit der DGVS vor. Allerdings muss darauf hingewiesen werden, dass der Kurs einem Basiskurs entspricht, der zunächst nur eine Fachkunde vermittelt. Praktische Fähigkeiten sollten im Anschluss daran, z. B. im Rahmen einer Hospitation, erarbeitet werden.

\section{Literatur}

1 Riphaus A, Wehrmann T, Weber B et al. S3-Leitlinie Sedierung in der gastrointestinalen Endoskopie. Z Gastroenterol 2008; 46: 1298-1330

2 Heuss LT, Schnieper P, Drewe J et al. Risk stratification and safe administration of propofol by registered nurses supervised by the gastroenterologist: a prospective observational study of more than 2000 cases. Gastrointest Endosc 2003; 57: 664-671

3 Rex DK, Overley C, Kinser K et al. Safety of propofol administered by registered nurses with gastroenterologist supervision in 2000 endoscopic cases. Am J Gastroenterol 2002; 97: 1159-1163 